\title{
CENTRALIZED RESOURCE ALLOCATION WITH THE POSSIBILITY OF DOWNSIZING IN TWO-STAGE NETWORK PRODUCTION SYSTEMS
}

\author{
Amir Hossein Yadolladi And Reza Kazemi Matin*i]
}

\begin{abstract}
The network data envelopment analysis (NDEA) technique has been recently developed to measure the relative efficiency of complex production systems. NDEA models provide more meaningful and informative results in comparison to the conventional black-box DEA approach that ignores the operations of the component processes. Regarding the centralized decision-making systems, normal management imposes common resource constraints to maximize produced outputs and minimize consumed inputs. The present study seeks to introduce new centralized resource allocation models in two-stage network production systems. This intra-organizational perspective also provides the possibility of closing down some of the existing units to improve system efficiency. To do so, three scenarios of centralized DEA models are introduced to take advantage of this possibility. A simple numerical example is used for illustration purposes. An empirical application of the proposed approach to the twenty branches of a university is also presented to show the applicability of the new approach.
\end{abstract}

Mathematics Subject Classification. 90B30.

Received March 26, 2021. Accepted July 31, 2021.

\section{INTRODUCTION}

The non-parametric frontier analysis was first introduced by Farrell [21] and later developed into Data Envelopment Analysis (DEA) by Charnes et al. [9] and Banker et al. [6], as a linear programming-based technique for assessing the efficiency of a set of homogenous decision-making units (DMUs). Nowadays, DEA is regarded as a well-known and powerful mathematical method for determining the relative efficiency of a set of functionally similar DMUs such as banks, brokerage firms, and insurance companies in a wide range of applications. Emrouznejad and Yang [17] provided an extensive survey of the first 40 years of scholarly literature in DEA.

Although the conventional DEA assumes that all DMUs benefit from complete autonomy in accessing available resources, the DMUs are often subjected to common resources and market constraints imposed by a central decision-maker in practice. The centralized decision-maker (DM) addresses a situation to monitor all the DMUs in many real-world scenarios. This type of situation occurs whenever all of the units belong to the same organization (public or private), which provides the units with the necessary resources to obtain their outputs. In such a decision-making environment, the centralized decision-maker aims to minimize the overall input consumption (or to maximize the overall output production) by all DMUs, instead of considering the consumption of each DMU separately $[15,18]$.

Keywords. Network data envelopment analysis, efficiency, centralized resource allocation, downsizing.

Department of Mathematics, Karaj Branch, Islamic Azad University, Karaj, Iran.

*Corresponding author: rkmatin@kiau.ac.ir

(c) The authors. Published by EDP Sciences, ROADEF, SMAI 2021 
In a centralized DM environment, a set of units operates under a central unit with the power to control certain decision parameters such as the allocation of resources to these units. To cope with such a situation, this research proposes a centralized DEA-based approach by considering the organizational setting. So far, various research has been conducted in this regard, including Athanassopoulos [3,4], Beasley [7], Färe et al. [19], Golany et al. [22] and Golany and Tamir [23]. Many authors have developed several centralized DEA-based models from different perspectives, which are input (output)-oriented under the constant or variable returns to scale technologies. In this regard, some approaches focus on minimizing the total input consumption by all the DMUs.

Korhonen and Syrjänen [26] developed a multiple-objective linear programming approach for resource allocation, aiming to maximize the total amount of outputs in all units, simultaneously. The approach was illustrated via a set of 20 fast-food restaurants in the city of Hefei, Anhui Province. Restaurants belonged to the same chain and had a central decision-making team consisting of several members who supervised the operations of all branches and design plans for future sales. Lozano and Villa [31] presented two centralized data envelopment analysis (CDEA) models to reduce the total quantity of resource consumed by all units in an organization, instead of considering the consumption of each unit separately. Asmild et al. [2] studied one of the centralized models proposed by Lozano and Villa [28] and modified it to only consider adjustments of previously inefficient units. Lozano et al. [30] implemented the centralized reallocation of emission allowances by using three objectives of maximizing aggregated desirable production, minimizing undesirable total emissions, and minimizing the consumption of input resources. Lozano et al. [31] proposed a centralized DEA approach to target setting and resource allocation in the Spanish national port system. Yu et al. [40] used a DEA-based aggregated concept to set up a modified centralized approach with a Russell measure to reallocate human resources. Mar-Molinero et al. [32] showed that the centralized resource allocation model can be substantially simplified and showed how the model works using real data of Spanish public schools. Recently, Song et al. [37] applied a DEA-based approach to consider production technology heterogeneity among DMUs, allocating a natural resource reduction amount to each DMU. Ripoll-Zarraga and Lozano [35] used a non-oriented slack-based inefficiency DEA model in order to reallocate two transferrable inputs (namely, labour costs and operating costs) between the different airports.

Most traditional DEA models, including the above-mentioned studies, mainly consider DMUs as black-boxes and ignore the internal structure of the production units [19]. In many practical situations, we encounter DMUs with multi-process structures, hereafter network structure, including intermediate products in which the outputs of the initial stage are used as inputs in the next stage. Therefore, considering black-box models for these structures may lead to overestimating the efficiency value of the units such that a unit is specified as an efficient one while it contains some inefficient components in its sub-processes. Seiford and Zhu [36] showed that considering banks as two-stage systems during the application of DEA in banking could lead to more reliable and accurate results.

Generally, two-stage production systems are reported as a basic and important category of such multiprocesses activities (e.g., refer to [10,38]). Up to now, a large number of studies have been carried out to deal with two-stage production systems and its extensions to more general cases from different points of view. Zhu [43] evaluated the efficiency scores of the best 500 companies by considering a two-stage structure. Lewis and Sexton [27] studied team performance in the Major Baseball League with two-stage DEA models. Färe et al. [20] introduced a method to analyze the performance of each sub-process, by considering intermediate products. Kao and Hwang [25] proposed standard DEA models by addressing the series relation between the stages of network systems as a straightforward extension of a two-stage case. Chen et al. [12] improved the method proposed by Korhonen and Syrjänen [26] to present efficient projections and benchmarking. Aviles-Sacoto et al. [5] considered a setting where the set of final outputs comprised the outputs of stage 2, as well as certain outputs from the previous stage. Zhang et al. [42] used a dynamic network slack-based measure (SBM) to study air pollution and health efficiency in Chinese cities and found that the production efficiency scores were slightly higher than the health efficiency scores, in addition to significant fluctuations in the two-stage efficiency scores of most cities. Patrizii [34] proposed a two-stage model with variable return to scale (VRS) and transformations to provide dual envelopment model. 
Chen et al. [12] considered DMUs with a two-stage network process that the shared input resources were used in both stages of operations. Also, they developed DEA models to measure the performance of two-stage network processes. Zha et al. [41] proposed a product-form cooperative efficiency model to demonstrate the overall efficiency of the DMU and the relationship between the stages, where the shared inputs can be freely allocated between two-stage production processes. Chen et al. [13] proposed a two-stage network DEA model to investigate the economic notion of economies of scope (ES) between two products. This paper has reviewed the related factors and offered a two-stage network CDEA model by integrating the two-stage NDEA and CDEA of resource allocation. Ang et al. [1] examined the allocation of resources to identify the cause of inefficiency of decision-making units in two-stage production systems.

Lozano and Villa [29] developed a procedure for efficiently allocating resources in a centralized decision-making setting, with the possibility to close down the operations of some DMUs in the interest of overall efficiency. Despite the existence of some centralized approaches in the literature, the possibility of closing or downsizing the DMUs is a novelty in DEA models. The present study focuses on modeling centralized resource allocation with the possibility of downsizing in the case of two-stage production networks. The main contributions of this paper are as follows:

- For the first time, a new approach is suggested to centralized resource allocation with the possibility of downsizing in the two-stage production system.

- A new non-radial Russell-based approach is proposed for discriminating strong and weak efficiencies.

- It is shown that deactivating some DMUs may be more effective in the centralized resource allocation in two-stage network production.

- Three different downsizing scenarios are suggested for allocating centralized resources and improving overall efficiency scores.

The rest of the paper is organized as follows. Section 2 provides a brief literature review on the existing twostage network DEA. Section 3 is devoted to introduce a new approach on the possibility of downsizing in the two-stage production system. In Section 4, numerical examples are utilized to explain the proposed approaches. Section 5 presents the application of the new two-stage centralized network DEA model for evaluating twenty branches of Islamic Azad University. Finally, Section 6 concludes the research.

\section{TWO-STAGE NETWORK}

So far, various approaches have been used to evaluate the efficiency of two-stage systems in conventional DEA models. In this study, the estimation of overall and divisional efficiency is generally performed using multiplier-based network models. A wide range of such models has been presented in network DEA literature. In specific, Kao and Hwang [25] calculated the efficiency of stage one under constant returns to scale (CRS) assumption. The overall efficiency score was then obtained by considering a series relationship between stages as the product of stage efficiencies. The intermediate products in both stages take equal weights, regardless of the role of intermediate products as outputs or inputs. Figure 1 illustrates a typical two-stage system.

As it is shown, $j=(1, \ldots, n), i=(1, \ldots, m)$, and $k=(1, \ldots, s)$ represent the indices for DMUs, inputs, and outputs, respectively, while $x_{i j} \in R_{+}$demonstrates the number of inputs $i$ consumed by $\mathrm{DMUj}, z_{g j} \in R_{+}$ $(g=1, \ldots, h)$ denotes the outputs of the first stage consumed as inputs for the second stage (intermediate products), and $y_{k j} \in R_{+}$is the quantity of output $k$ produced by $\mathrm{DMU}_{j}$. Moreover, we assume that observed inputs, intermediate products, and output vectors are non-negative with at least one positive component. In addition, notations $\theta$ and $\varphi$ are used for the radial contraction of the total input vector and the total output vector, respectively. Finally, the parameters $\lambda_{j}^{1}=\left(\lambda_{1}^{1}, \lambda_{2}^{1}, \ldots, \lambda_{n}^{1}\right)$ and $\lambda_{j}^{2}=\left(\lambda_{1}^{2}, \lambda_{2}^{2}, \ldots, \lambda_{n}^{2}\right)$ are the vectors of non-negative intensity weights for stage one and stage two corresponding to $\mathrm{DMU}_{j}$, respectively.

Kao and Hwang [25] considered a two-stage serial technology with sub-technologies connected to form a network with the VRS assumption, and proposed an envelopment output-oriented model as follows: 


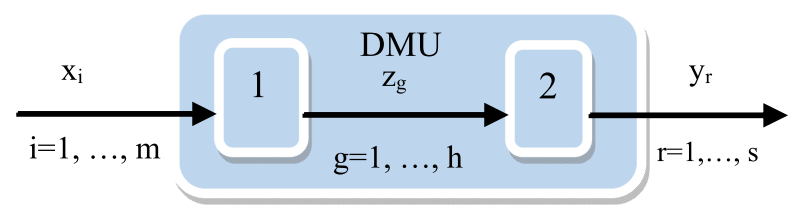

Figure 1. A simple two-stage production system.

$$
\begin{aligned}
& \operatorname{Max}_{\varphi, \lambda} \varphi \\
& \text { s.t. } \sum_{j=1}^{n} \lambda_{j}^{1} x_{i j} \leq x_{i o} \\
& i=1, \ldots, m \\
& \sum_{j=1}^{n} \lambda_{j}^{1} z_{g j} \geq \sum_{j=1}^{n} \lambda_{j}^{2} z_{g j} \\
& g=1, \ldots, h \\
& \begin{array}{l}
\sum_{j=1}^{n} \lambda_{j}^{2} y_{k j} \geq \\
\sum_{j=1}^{n} \lambda_{j}^{1}=1
\end{array} \\
& \sum_{j=1}^{n} \lambda_{j}^{2}=1 \\
& \lambda_{j}^{1} \geq 0, \lambda_{j}^{2} \geq 0 \\
& k=1, \ldots, s \\
& j=1, \ldots, n .
\end{aligned}
$$

According to the model (2.1), in the following section, we present the "centralized resource allocation with the possibility of downsizing" as a novel idea.

\section{Evaluating TWO-STAGE NETWORK SYSTEMS WITH THE POSSIBILITY OF DOWNSIZING}

In this section, the idea of centralized resource allocation with the possibility of downsizing by Lozano and Villa [29] is expanded for two-stage production networks. To evaluate the efficiency, the Russell-based model introduced by Pastor et al. [33] is considered to provide Pareto-Koopmans efficient criteria in the evaluation, by individually reducing the sum of inputs and increasing the sum of outputs.

Let $\theta_{i}$ denote radial contraction of the total amount of $i$ th input and $\varphi_{k}$ represent the radial contraction of the total amount of $k$ th output, and $\lambda_{r}^{1}=\left(\lambda_{1 r}^{1}, \lambda_{2 r}^{1}, \ldots, \lambda_{n r}^{1}\right)$ and $\lambda_{r}^{2}=\left(\lambda_{1 r}^{2}, \lambda_{2 r}^{2}, \ldots, \lambda_{n r}^{2}\right)$ are the vectors of non-negative intensity weights for stage one and stage two for projecting $\mathrm{DMU}_{r}$. Also, $\delta_{r}=\left\{\begin{array}{ll}1 & \text { if } \mathrm{DMU}_{r} \text { is active } \\ 0 & \text { if } \mathrm{DMU}_{r} \text { is not active }\end{array}\right.$.

Using these notions, the following three scenarios can be addressed for resource reallocation, while considering the possibility of downsizing for two-stage network production systems:

- Scenario (i): flexible inputs/outputs improvement with flexible downsizing criteria.

- Scenario (ii): flexible inputs/outputs improvement with fixed downsizing criteria.

- Scenario (iii): fixed inputs/outputs improvement with flexible downsizing criteria.

\subsection{Scenario (i): reducing inputs and increasing outputs with flexible downsizing criteria}

This scenario presents a non-radial centralized resource allocation model for a two-stage network system, in which maintaining the active status for all existing units is not required. The main purpose of discussing this 
scenario is to study the intra-organizational performance of production systems for calculating new operating points, while allowing for some deactivated decision-making units. The possibility of reducing the number of the existing DMUs is included in the model by allowing them to be projected onto a virtual empty operating point that takes no inputs and produces no outputs. Deactivating some decision-making units may be beneficial to the whole organization since the total amount of reduced inputs can be allocated among the remaining active decision-making units, following by an increase in the total produced outputs. In this scenario, the model decides to deactivate some decision units to improve the production system; however, it is likely for the unit not to be deactivated in this scenario. The possibility of reducing the number of the observed active DMUs is included in this model by inserting binary variables $\delta_{r}$.

$$
\begin{aligned}
& \underset{\theta, \varphi, \lambda, \delta}{\operatorname{Min}} \frac{\frac{1}{m} \sum_{i=1}^{m} \theta_{i}}{\frac{1}{s} \sum_{k=1}^{s} \varphi_{k}} \\
& \text { s.t. } \sum_{r=1}^{n} \sum_{j=1}^{n} \lambda_{j r}^{1} x_{i j} \leq \theta_{i} \sum_{r=1}^{n} x_{i r} \quad i=1, \ldots, m \\
& \sum_{r=1}^{n} \sum_{j=1}^{n} \lambda_{j r}^{1} z_{g j} \geq \sum_{r=1}^{n} \sum_{j=1}^{n} \lambda_{j r}^{2} z_{g j} \quad g=1, \ldots, h \\
& \sum_{r=1}^{n} \sum_{j=1}^{n} \lambda_{j r}^{2} y_{k j} \geq \varphi_{k} \sum_{r=1}^{n} y_{k r} \quad k=1, \ldots, s \\
& \sum_{j=1}^{n} \lambda_{j r}^{1}=\delta_{r} \quad r=1, \ldots, n \\
& \sum_{j=1}^{n} \lambda_{j r}^{2}=\delta_{r} \quad r=1, \ldots, n \\
& \lambda_{j r}^{1}, \lambda_{j r}^{2} \geq 0 \quad j, r=1, \ldots, n \\
& 0 \leq \theta_{i} \leq 1, \varphi_{k} \geq 1 \quad i=1, \ldots, m, k=1, \ldots, s \\
& \delta_{r} \in\{0,1\} \quad r=1, \ldots, n .
\end{aligned}
$$

This model has a fractional objective with linear constraints and binary variables. The first constraint regards decreasing the total of inputs available in all production units, the second constraint relates to intermediate products, and the third constraint considers the increasing criteria in the total of outputs for all production units. Note that $\left(\forall i: \theta_{i}=1, \forall k: \varphi_{k}=1, \lambda_{r r}^{1}=\lambda_{r r}^{2}=\delta_{r}=1, \forall j \neq r: \lambda_{j r}^{1}=\lambda_{j r}^{2}=0\right)$ satisfies all constraints of this model; so model (3.1) is feasible and the objective function is bounded.

After solving this model, the optimal values for $\theta_{i}^{*}$ and $\varphi_{k}^{*}$ are obtained, which represent the optimal scale factors for the total of each input and output, respectively. Moreover, $N^{*}=\sum_{r=1}^{n} \delta_{r}^{*}$ indicates the total number of operating units to optimize the overall system performance. The set of optimal vectors of multipliers for stages 1 and 2 is $\left(\lambda_{1 r}^{1^{*}}, \lambda_{2 r}^{1^{*}}, \ldots, \lambda_{m r}^{1^{*}}\right)$ and $\left(\lambda_{1 r}^{2^{*}}, \lambda_{2 r}^{2^{*}}, \ldots, \lambda_{m r}^{2^{*}}\right)$, which defines each optimal (benchmark) operating unit. The input and output targets corresponding to these operating points can be calculated as follows:

For the first stage, $\forall i: \hat{x}_{i r}=\sum_{j=1}^{n} \lambda_{j r}^{1^{*}} x_{i j}, \forall g: \hat{z}_{g r}^{\text {out }}=\sum_{j=1}^{n} \lambda_{j r}^{1^{*}} z_{g j}$, and for the second stage, $\forall g: \hat{z}_{g r}^{\text {in }}=$ $\sum_{j=1}^{n} \lambda_{j r}^{2^{*}} z_{g j}, \forall k: \hat{y}_{k r}=\sum_{j=1}^{n} \lambda_{j r}^{2^{*}} y_{k j}$.

Remark 3.1. It is easy to verify that any unit that is deactivated in stage one, will also be deactivated in stage two. In this case, there are no inputs to produce the intermediate products and, therefore the final outputs cannot be produced as well.

\subsubsection{Linearization}

The model in (3.1) has a fractional programming structure. Therefore, we can utilize the approach proposed in Charnes and Cooper [8] to transform the nonlinear model in (3.1) into an equivalent linear constrained 
programming formulation, which can readily be solved. To do so, we introduce a new variable, $\beta$, defined by $\beta=\left(\sum_{r=1}^{s} \frac{\varphi_{r}}{s}\right)^{-1}$ such that $0<\beta \leq 1$ and $\beta\left(\sum_{r=1}^{s} \frac{\varphi_{r}}{s}\right)=1$. Then, we use $\beta$ to transform the variables from (3.1) into new variables defined by $\theta_{i}^{\prime}=\beta \theta_{i}(i=1, \ldots, m), \varphi_{k}^{\prime}=\beta \varphi_{k}(k=1, \ldots, s), \lambda_{j r}^{1^{\prime}}=\beta \lambda_{j r}^{1}(j, r=1, \ldots, n)$, $\lambda_{j r}^{2^{\prime}}=\beta \lambda_{j r}^{2}(j, r=1, \ldots, n)$, and $\delta_{r}^{\prime}=\beta \delta_{r}(r=1, \ldots, n)$. We can then multiply the numerator and the denominator in the objective of (3.1) by $\beta$ without changing its value. Moreover, since $\beta>0$, we can multiply both sides of the constraints in (3.1) by this variable without changing any of the orientations. Finally, we then write the transformed model as follows:

$$
\begin{aligned}
& \operatorname{Min}_{\theta^{\prime}, \varphi^{\prime}, \lambda^{\prime}, \delta^{\prime}} \frac{1}{m} \sum_{i=1}^{m} \theta_{i}^{\prime} \\
& \text { s.t. } \sum_{k=1}^{s} \varphi_{k}^{\prime}=s \\
& \sum_{r=1}^{n} \sum_{j=1}^{n} \lambda_{j r}^{1^{\prime}} x_{i j} \leq \theta_{i}^{\prime} \sum_{r=1}^{n} x_{i r} \quad i=1, \ldots, m \\
& \sum_{r=1}^{n} \sum_{j=1}^{n} \lambda_{j r}^{1^{\prime}} z_{g j} \geq \sum_{r=1}^{n} \sum_{j=1}^{n} \lambda_{j r}^{2^{\prime}} z_{g j} \quad g=1, \ldots, h \\
& \sum_{r=1}^{n} \sum_{j=1}^{n} \lambda_{j r}^{2^{\prime}} y_{k j} \geq \varphi_{k}^{\prime} \sum_{r=1}^{n} y_{k r} \quad k=1, \ldots, s \\
& \sum_{j=1}^{n} \lambda_{j r}^{1^{\prime}}=\delta_{r}^{\prime} \quad r=1, \ldots, n \\
& \sum_{j=1}^{n} \lambda_{j r}^{2^{\prime}}=\delta_{r}^{\prime} \quad r=1, \ldots, n \\
& \lambda_{j r}^{1^{\prime}}, \lambda_{j r}^{2^{\prime}} \geq 0 \quad j, r=1, \ldots, n \\
& \theta_{i}^{\prime} \leq \beta, \varphi_{k}^{\prime} \geq \beta \quad i=1, \ldots, m, k=1, \ldots, s \\
& \delta_{r}^{\prime}=\left(1-t_{r}\right) \beta \quad r=1, \ldots, n \\
& t_{r} \in\{0,1\} .
\end{aligned}
$$

Note that the new model has linear structure in objective function and all the constraints, except the last one; in which the binary variable $t_{r}$ is exists. This model could be easily solved with Branch-and-Bound (B\&B) algorithms for obtaining the global optimal solutions. A similar linearization approach can be stated for the other scenarios.

\subsection{Scenario (ii): reducing inputs and increasing outputs with fixed downsizing criteria}

In this scenario, a limited number of active DMUs are considered to maximize the overall efficiency by reducing the total inputs and increasing the total outputs. The number of active units in this scenario is determined by the decision maker, i.e., the decision maker deactivates some decision units to improve the state of the production system. However, the process of determining which decision units remain active and which units are disabled is performed by the model. In this case, we try to improve the overall performance of the production system based on this limitation. It should be noted that the decision maker also faces a limitation in choosing the number of active decision units, which will be examined in a separate scenario. Let $N^{\text {target }}<n$ represent the maximum desired number of surviving DMUs.

$$
\underset{\theta, \varphi, \lambda, \delta}{\operatorname{Min}} \frac{\frac{1}{m} \sum_{i=1}^{m} \theta_{i}}{\frac{1}{s} \sum_{k=1}^{s} \varphi_{k}}
$$




$$
\begin{aligned}
& \text { s.t. } \sum_{r=1}^{n} \sum_{j=1}^{n} \lambda_{j r}^{1} x_{i j} \leq \theta_{i} \sum_{r=1}^{n} x_{i r} \quad i=1, \ldots, m \\
& \sum_{r=1}^{n} \sum_{j=1}^{n} \lambda_{j r}^{1} z_{g j} \geq \sum_{r=1}^{n} \sum_{j=1}^{n} \lambda_{j r}^{2} z_{g j} \quad g=1, \ldots, h \\
& \sum_{r=1}^{n} \sum_{j=1}^{n} \lambda_{j r}^{2} y_{k j} \geq \varphi_{k} \sum_{r=1}^{n} y_{k r} \quad k=1, \ldots, s \\
& \sum_{j=1}^{n} \lambda_{j r}^{1}=\delta_{r} \quad r=1, \ldots, n \\
& \sum_{j=1}^{n} \lambda_{j r}^{2}=\delta_{r} \quad r=1, \ldots, n \\
& \sum_{r=1}^{n} \delta_{r} \leq N^{\text {target }} \\
& \lambda_{j r}^{1}, \lambda_{j r}^{2} \geq 0 \\
& \theta_{i} \leq 1, \varphi_{k} \geq 1 \\
& \delta_{r} \in\{0,1\} \\
& \begin{array}{l}
j, r=1, \ldots, n \\
i=1, \ldots, m, k=1, \ldots, s \\
r=1, \ldots, n
\end{array}
\end{aligned}
$$

This model is similar to model (3.1), with the exception of an extra constraint $\sum_{r=1}^{n} \delta_{r} \leq N^{\text {target }}$, which guarantees the maximum number of active units in the DM's view. Note that this model is also feasible and bounded. A similar linearization approach, discussed for scenario (i), should be considered in the computations stage of this model. For the new constraint, by multiplying with $\beta$ we have $\sum_{r=1}^{n} \delta_{r}^{\prime} \leq \beta N^{\text {target}}$. Now by solving model (3.3), we obtain a set of optimal values for variables $\theta_{i}^{*}, \varphi_{k}^{*}, \sum_{r=1}^{n} \delta_{r}^{*} \leq N^{\text {target }},\left(\lambda_{1 r}^{1^{*}}, \lambda_{2 r}^{1^{*}}, \ldots, \lambda_{m r}^{1^{*}}\right)$ and $\left(\lambda_{1 r}^{2^{*}}, \lambda_{2 r}^{2^{*}}, \ldots, \lambda_{m r}^{2^{*}}\right)$.

\subsection{Scenario (iii): fixed improvement in the total inputs and/or outputs with flexible downsizing criteria}

In this section, a model is proposed to obtain the specified reduction in the total amount of available inputs, along with the specified increase in the total amount of produced outputs, to reduce the number of operating units as much as possible. If the decision maker wants to achieve a specific reduction in the present number of total inputs and a specific increase in the present number of total outputs to improve the overall performance of the production system, it must reduce the number of operating units as much as possible. So, the purpose here is to minimize the number of operating units. To this end, we suggest a two-step calculation.

The first step minimizes the number of surviving units, while the total number of inputs and outputs are optimized in the second step. Let $\hat{\theta}_{i} \leq 1$ and $\hat{\varphi}_{k} \geq 1$ denote the DM-suggested value for improvements in the total amount of $i$ th input and $k$ th output, respectively. The following model is suggested to find the minimum surviving units with these values.

$$
\begin{aligned}
& \operatorname{Min}_{\lambda, \delta} \sum_{r=1}^{n} \delta_{r} \\
& \text { s.t. } \sum_{r=1}^{n} \sum_{j=1}^{n} \lambda_{j r}^{1} x_{i j} \leq \hat{\theta}_{i} \sum_{r=1}^{n} x_{i r} \quad i=1, \ldots, m \\
& \sum_{r=1}^{n} \sum_{j=1}^{n} \lambda_{j r}^{1} z_{g j} \geq \sum_{r=1}^{n} \sum_{j=1}^{n} \lambda_{j r}^{2} z_{g j} \quad g=1, \ldots, h
\end{aligned}
$$




$$
\begin{array}{ll}
\sum_{r=1}^{n} \sum_{j=1}^{n} \lambda_{j r}^{2} y_{k j} \geq \hat{\varphi}_{k} \sum_{r=1}^{n} y_{k r} & k=1, \ldots, s \\
\sum_{j=1}^{n} \lambda_{j r}^{1}=\delta_{r} & r=1, \ldots, n \\
\sum_{j=1}^{n} \lambda_{j r}^{2}=\delta_{r} & r=1, \ldots, n \\
\lambda_{j r}^{1}, \lambda_{j r}^{2} \geq 0 & j, r=1, \ldots, n \\
\delta_{r} \in\{0,1\} & r=1, \ldots, n .
\end{array}
$$

It should be noted that it is possible to obtain an infeasible case using the above optimization model. In this case, initial DM parameters should be modified. On the other hand, for the case of a feasible solution, it is easy to show that there exists at least one surviving unit as the optimal solution. Otherwise, if $\sum_{r=1}^{n} \delta_{r}^{*}=0$, then according to the fifth set of constraints, we have $\sum_{j=1}^{n} \lambda_{j r}^{* 2}=0$ for all observed units. So, the third set of constraints yields to $\hat{\varphi}_{k} \sum_{r=1}^{n} y_{k r} \leq 0$. This is an obvious contradiction with the basic assumptions of $\hat{\varphi}_{k} \geq 1$ and $\sum_{r=1}^{n} y_{k r}>0$.

Let the natural number $N^{*}$, defined by $\sum_{r=1}^{n} \delta_{r}^{*}=N^{*}$, show the optimal value for the objective function in the model (3.4). By maintaining this number for active units, the next step is suggested to improve the total of inputs and outputs.

$$
\begin{aligned}
& \underset{\theta, \varphi, \lambda, \delta}{\operatorname{Min}} \frac{\frac{1}{m} \sum_{i=1}^{m} \theta_{i}}{\frac{1}{s} \sum_{k=1}^{s} \varphi_{k}} \\
& \text { s.t. } \sum_{r=1}^{n} \sum_{j=1}^{n} \lambda_{j r}^{1} x_{i j} \leq \theta_{i} \sum_{r=1}^{n} x_{i r} \quad i=1, \ldots, m \\
& \sum_{r=1}^{n} \sum_{j=1}^{n} \lambda_{j r}^{1} z_{g j} \geq \sum_{r=1}^{n} \sum_{j=1}^{n} \lambda_{j r}^{2} z_{g j} \quad g=1, \ldots, h \\
& \sum_{r=1}^{n} \sum_{j=1}^{n} \lambda_{j r}^{2} y_{k j} \geq \varphi_{k} \sum_{r=1}^{n} y_{k r} \quad k=1, \ldots, s \\
& \sum_{j=1}^{n} \lambda_{j r}^{1}=\delta_{r} \quad r=1, \ldots, n \\
& \sum_{j=1}^{n} \lambda_{j r}^{2}=\delta_{r} \quad r=1, \ldots, n \\
& \sum_{r=1}^{n} \delta_{r}=N^{*} \\
& \lambda_{j r}^{1}, \lambda_{j r}^{2} \geq 0 \quad j, r=1, \ldots, n \\
& \theta_{i} \leq 1, \varphi_{k} \geq 1 \quad i=1, \ldots, m, k=1, \ldots, s \\
& \delta_{r} \in\{0,1\} \quad r=1, \ldots, n .
\end{aligned}
$$

Solving this model yields the following set of similar information regarding the scenario: optimal values for variables $\theta_{i}^{*}, \varphi_{k}^{*}, N^{*}=\sum_{r=1}^{n} \delta_{r}^{*},\left(\lambda_{1 r}^{1 *}, \lambda_{2 r}^{1 *}, \ldots, \lambda_{m r}^{1 *}\right)$, and $\left(\lambda_{1 r}^{2 *}, \lambda_{2 r}^{2 *}, \ldots, \lambda_{m r}^{2 *}\right)$, along with input and output targets corresponding to the optimal operating benchmark.

In the next section, we present an illustrative example by considering the possibility of downsizing to discuss three scenarios further and compare the results with the traditional radial measurements. 
TABLE 1. Data of seven DMUs.

\begin{tabular}{llll}
\hline \hline DMU & $x$ & $z$ & $y$ \\
\hline $\mathrm{A}$ & 1 & 1 & 1 \\
$\mathrm{~B}$ & 2 & 1 & 0.5 \\
$\mathrm{C}$ & 1 & 1.5 & 2 \\
$\mathrm{D}$ & 2 & 3 & 4 \\
$\mathrm{E}$ & 2 & 4 & 8 \\
$\mathrm{~F}$ & 3 & 3 & 3 \\
$\mathrm{G}$ & 2 & 4 & 7 \\
\hline Total & 13 & 17.5 & 25.5 \\
\hline
\end{tabular}

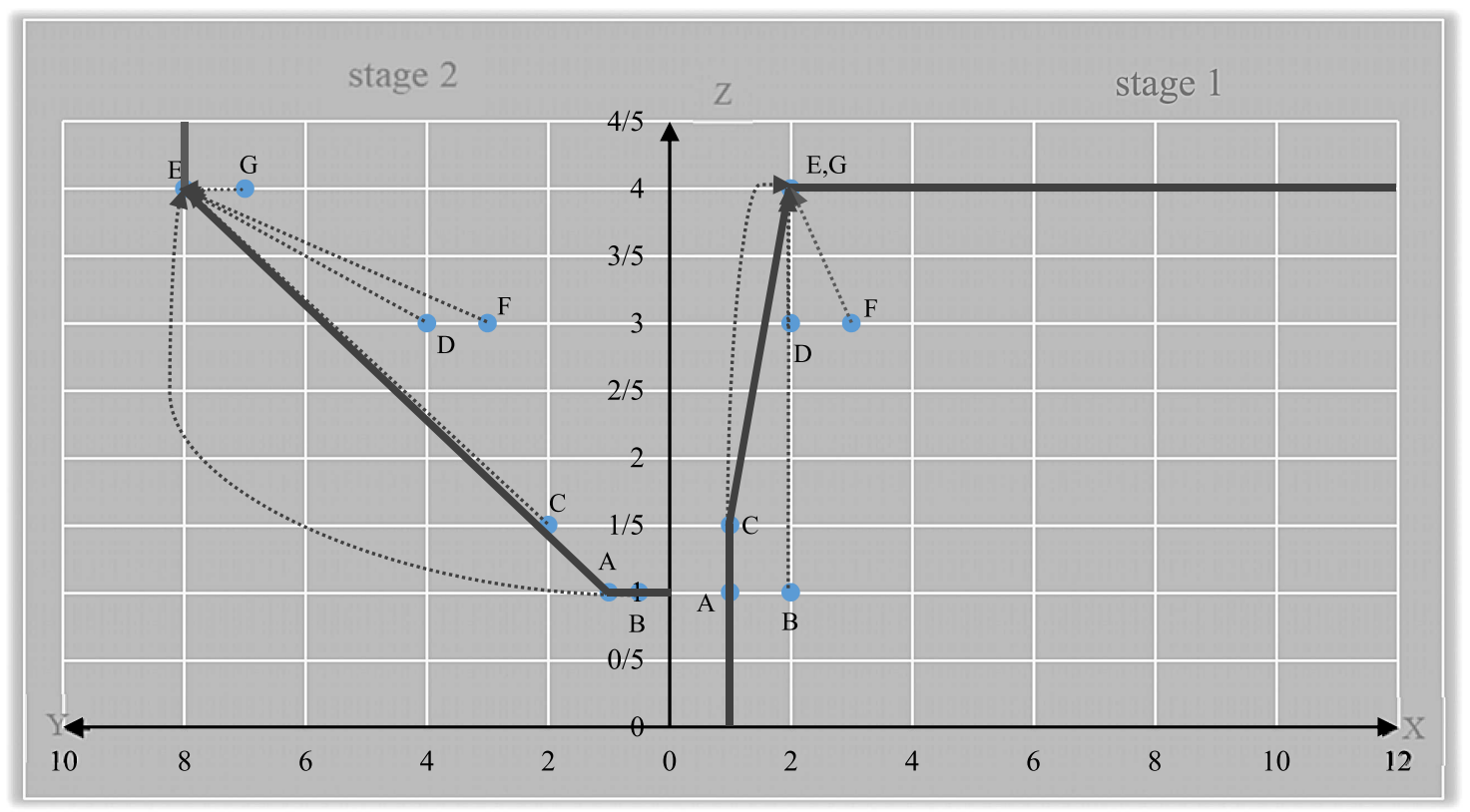

Figure 2. Production possibility sets (stages 1 and 2) and project points.

\section{Illustrative eXAmple}

In this section, we present a numerical example for a simple two-stage production network using the introduced approach. Table 1 reports the data.

For illustration purposes, we will now proceed with implementing the model presented in the first scenario, along with its details and optimal solution. Figure 2 shows the production set associated with the sub-processes for the first scenario.

The results for an efficiency analysis on the two-stage production system using scenario (i) are reported in Table 2. This scenario reduces the total inputs obtained by the proposed approach by $8 \%$ and increases the total outputs by $88 \%$. It is worth noting that one of the observed units is closed, and the objective function values are $\theta^{*}=0.923$ and $\varphi^{*}=1.882$.

In the first stage, points $\mathrm{C}, \mathrm{E}$, and $\mathrm{G}$ are technically efficient frontiers, while point $\mathrm{A}$ is the weak efficient frontier. In contrast, points $\mathrm{D}, \mathrm{B}$, and $\mathrm{F}$ are inefficient in this stage. After improving inputs and outputs of the 
TABLE 2. Computed benchmarks in the three scenarios.

\begin{tabular}{|c|c|c|c|c|c|c|c|c|c|c|c|c|}
\hline \multirow[b]{2}{*}{$\mathrm{DMU}$} & \multicolumn{4}{|c|}{ Scenario (i) } & \multicolumn{4}{|c|}{ Scenario (ii) } & \multicolumn{4}{|c|}{ Scenario (iii) } \\
\hline & $\hat{x}$ & $z^{\text {out }}$ & $\hat{z}^{\text {in }}$ & $\hat{y}$ & $\hat{x}$ & $z^{\text {out }}$ & $\hat{z}^{\text {in }}$ & $\hat{y}$ & $\hat{x}$ & $z^{\text {out }}$ & $\hat{z}^{\text {in }}$ & $\hat{y}$ \\
\hline $\mathrm{A}$ & 0 & 0 & 0 & 0 & 2 & 4 & 4 & 8 & 0 & 0 & 0 & 0 \\
\hline B & 2 & 4 & 4 & 8 & 2 & 4 & 4 & 8 & 0 & 0 & 0 & 0 \\
\hline $\mathrm{C}$ & 2 & 4 & 4 & 8 & 2 & 4 & 4 & 8 & 0 & 0 & 0 & 0 \\
\hline $\mathrm{D}$ & 2 & 4 & 4 & 8 & 0 & 0 & 0 & 0 & 2 & 4 & 4 & 8 \\
\hline $\mathrm{E}$ & 2 & 4 & 4 & 8 & 2 & 4 & 4 & 8 & 2 & 4 & 4 & 8 \\
\hline $\mathrm{F}$ & 2 & 4 & 4 & 8 & 0 & 0 & 0 & 0 & 2 & 4 & 4 & 8 \\
\hline $\mathrm{G}$ & 2 & 4 & 4 & 8 & 2 & 4 & 4 & 8 & 2 & 4 & 4 & 8 \\
\hline \multirow[t]{2}{*}{ Total } & 12 & 24 & 24 & 48 & 10 & 20 & 20 & 40 & 8 & 16 & 16 & 32 \\
\hline & & & & & & $N^{\text {targ }}$ & $=4$ & & $\sigma$ & $=0.8$ & $b_{k}=$ & \\
\hline
\end{tabular}

units, point $\mathrm{A}$ is inactive, while other units are projected on the optimal points $\mathrm{E}$ and $\mathrm{G}$. In the second stage, points $\mathrm{A}$ and $\mathrm{E}$ are on the technically efficient part of the frontier, point $\mathrm{B}$ is on the weak efficient frontier, and points $\mathrm{D}, \mathrm{G}$, and $\mathrm{F}$ are inefficient. Therefore, inefficient points are improved and projected on the point E. In this stage, unit A is once again inactive, while other units are projected on point $\mathrm{E}$.

All the introduced scenarios are examined in this numerical example. Table 2 represents the solution obtained using the non-radial two-stage network with the possibility of downsizing.

The result shows a reduction of approximately $23 \%$ in the total consumed inputs, which was obtained using the approach proposed in the scenario (ii). Note that the results for this scenario are achieved with $N^{\text {target }}=5$, indicating that at least two of the observed DMUs should be closed. Since this result means closing one unit more than the optimal result obtained in the scenario (i), a higher reduction in the total input is about $\frac{0.923-0.769}{0.923} \times$ $100 \cong 16 \%$ along with an increase of approximately $56 \%$ in the total produced outputs occurs. In this scenario, the objective function values are $\theta^{*}=0.769$ and $\varphi^{*}=1.569$. Note that this result is highly dependent on the selected target number for active units, i.e., $N^{\text {target }}$. For instance, the scenario (ii) is infeasible with any value less than $N^{\text {target }}=4$.

Regarding the result of the scenario (iii), the DM decides to save $20 \%$ on the total input and increase the total output by $20 \%$. With this information, three units were closed, indicating that this scenario, compared with the optimal result from the scenario (ii), increases the terminating number of units by one. The objective function values are $\theta^{*}=0.615$ and $\varphi^{*}=1.255$.

\section{EMPIRICAL APPLICATION}

Assessing efficiency is an important issue in higher education institutions and universities. During recent decades, DEA techniques have been widely used in educational applications by considering educational institutions, universities, faculties, departments, and programs as DMUs. Network DEA models have also been used in assessment of higher education institutions, from different point of views. See for example Yang et al. [39] who presented a general two-stage network for assessing 64 Chinese research universities and their evolution over the period of 2010-2013. Hosseini et al. [24] analyzed the regress and progress of educational performance of some universities by applying a general network DEA approach with stochastic data. More recently, Ding et al. [14] proposed an additive DEA model to evaluate the overall efficiency of the non-homogeneous DMUs with a two-stage network structure with application in performance assessment of some university departments in China.

In this section, using the new technique of allocating resources in such a way that allows the potential deactivation of some decision-making units, we will try to improve the overall performance of twenty branches of Islamic Azad University. Most of the studies in the relevant literature focus on analyzing the performance 


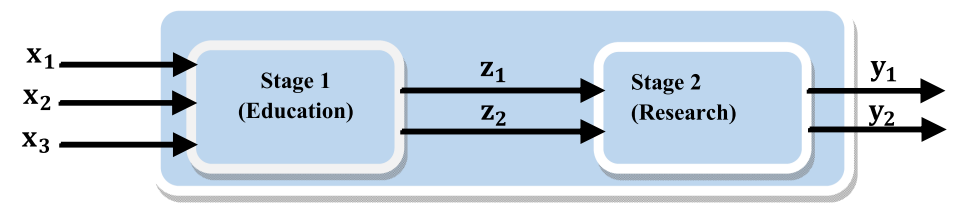

FiguRE 3. Two stage network structure of activities in a higher education institution.

TABLE 3. Total amounts of inputs, intermediate products, and outputs for the 20 DMUs.

\begin{tabular}{llllllll}
\hline \hline & $x_{1}$ & $x_{2}$ & $x_{3}$ & $z_{1}$ & $z_{2}$ & $y_{1}$ & $y_{2}$ \\
\hline Total & 2462 & 217578 & 3406 & 110751 & 29247.77 & 8071.64 & 55800 \\
\hline
\end{tabular}

in terms of teaching and/or research from a variety of perspectives. It should be noted that the IAU campus branches are comprised of different departments. The two major management offices in the campuses include the Education Department (stage 1), and the Research Department (stage 2). These departments cooperate in the network system, as depicted in Figure 3. The Education Department is responsible for graduating students from predefined courses, while having non-academic personnel, academic personnel, and a number of students as the main factors with the aim of generating income (tuition) and graduates. The Research Department provides the graduate students with its products, including published journal articles, conference papers, books, and so on. The final outputs from these two departments include the number of research products and total university income. The total inputs, the total intermediates, and the total outputs used in this evaluation are collected for the educational year 2015 from ISCS ${ }^{1}$ unit, are presented in Table 3.

\section{Inputs}

$x_{1}$ : Number of employees

$x_{2}$ : Number of students

$x_{3}$ : Number of faculty members

\section{Intermediate products}

$z_{1}$ : Number of graduates

$z_{2}$ : Tuition fees (in billion Rialls)

\section{Outputs}

$y_{1}$ : Total university income (in billion Rialls)

$y_{2}$ : Number of research products

Now, we implement the three scenarios presented in this study on this application. We used Lingo 17 software on an Intel Corei5, 4G bytes RAM, $2.4 \mathrm{GHz}$ Laptop. The computational times for the new introduced models were just a few seconds per MILP problem.

Table 4 presents the results for scenario (i). As can be seen, the total reduction for the first, second and third inputs are $80 \%, 69 \%$ and $42 \%$, respectively. These reductions are obtained while maintaining the two outputs. Besides, as can be seen, seven units have been deactivated; i.e., U1, U2, U9, U10, U12, U14 and U20 are deactivated units in this scenario. The intermediate products, including the number of graduates and the tuition fees, which are also considered as the products of the education stage, have reached the values of 80777.75 and 40373.91 , respectively. Given that these products of the educational stage are used as inputs for

\footnotetext{
${ }^{1}$ Information and statistics collection system (https://stat.iau.ir/).
} 
TABLE 4. The results for the scenario (i).

\begin{tabular}{llllllllll}
\hline \hline & $\hat{x}_{1}$ & $\hat{x}_{2}$ & $\hat{x}_{3}$ & $\hat{z}_{1}^{\text {out }}$ & $\hat{z}_{1}^{\text {in }}$ & $\hat{z}_{2}^{\text {out }}$ & $\hat{z}_{2}^{\text {in }}$ & $\hat{y}_{1}$ & $\hat{y}_{2}$ \\
\hline U1 & 0 & 0 & 0 & 0 & 0 & 0 & 0 & 0 & 0 \\
U2 & 0 & 0 & 0 & 0 & 0 & 0 & 0 & 0 & 0 \\
U3 & 35 & 4945 & 164 & 6716 & 9327 & 3146.3 & 701.9 & 770.7 & 4879 \\
U4 & 36.4 & 5059.8 & 159.44 & 6494.75 & 5943.16 & 3128.41 & 5362.67 & 753.48 & 4132.82 \\
U5 & 35 & 4945 & 164 & 6716 & 407 & 3146.3 & 2636.2 & 640.9 & 2923 \\
U6 & 35 & 4945 & 164 & 6716 & 407 & 3146.3 & 2636.2 & 640.9 & 2923 \\
U7 & 35 & 4945 & 164 & 6716 & 9327 & 3146.3 & 701.9 & 770.7 & 4879 \\
U8 & 75 & 8221 & 34 & 407 & 1487 & 2636.2 & 5.07 & 7.14 & 3682 \\
U9 & 0 & 0 & 0 & 0 & 0 & 0 & 0 & 0 & 0 \\
U10 & 0 & 0 & 0 & 0 & 0 & 0 & 0 & 0 \\
U11 & 35 & 4945 & 164 & 6716 & 9327 & 3146.3 & 701.9 & 770.7 & 4879 \\
U12 & 0 & 0 & 0 & 0 & 0 & 0 & 0 & 0 & 0 \\
U13 & 35 & 4945 & 164 & 6716 & 1487 & 3146.3 & 5.07 & 7.14 & 3682 \\
U14 & 0 & 0 & 0 & 0 & 0 & 0 & 0 & 0 \\
U15 & 35 & 4945 & 164 & 6716 & 890.38 & 3146.3 & 1458.56 & 459.77 & 4337.17 \\
U16 & 35 & 4945 & 164 & 6716 & 9310 & 3146.3 & 7577.6 & 826.5 & 4868 \\
U17 & 35 & 4945 & 164 & 6716 & 9310 & 3146.3 & 7577.6 & 826.5 & 4868 \\
U18 & 35 & 4945 & 164 & 6716 & 9327 & 3146.3 & 701.9 & 770.7 & 4879 \\
U19 & 35 & 4945 & 164 & 6716 & 9310 & 3146.3 & 7577.6 & 826.5 & 4868 \\
U20 & 0 & 0 & 0 & 0 & 0 & 0 & 0 & 0 \\
\hline Total & 496.4 & 67675.8 & 1997.4 & 80777.75 & 75859.54 & 40373.91 & 37644.17 & 8071.6 & 55800 \\
\hline Optimal & $\theta^{*}=0.201$ & $\theta^{*}=0.311$ & $\theta^{*}=0.5864$ & - & - & - & - & $\varphi^{*}=1$ & $\varphi^{*}=1$ \\
\hline
\end{tabular}

the research stage, in this scenario, 75859.54 students graduated and the existing tuition of 37644.17 , has been used as input for the research stage.

Next, we consider the report according to the scenario (ii).

Table 5 presents the results of scenario (ii). In this scenario, it is assumed that the decision maker's purpose is to allocate resources by deactivating three DMUs among the Islamic Azad University units, i.e., the results of this scenario are achieved with $N^{\text {target }}=15$. As can be seen, the total reduction for the first, second and third inputs are $66 \%, 54 \%$, and $57 \%$, respectively. These reductions are again obtained while maintaining the two outputs. The intermediate products include the number of graduates and the tuition fees, which are also considered as the products of the education stage, and they have reached the values of 51960.23 and 43250.52 , respectively. Given that the products of the educational stage are used as inputs for the research stage, in this scenario, 51960.23 students graduated and the existing tuition of 43249.8 has been used as input for the research stage.

Furthermore, let us consider the example according to the scenario (iii).

As Table 6 indicates, in this scenario, it is assumed that the DM decides to save $40 \%\left(\hat{\theta}_{1}=0.6\right)$ on the total amount of the first input, and $40 \%\left(\hat{\theta}_{3}=0.6\right)$ on the total of the third input. In this case, 8 operating units have been deactivated. Based on the decreases attained by the DM for the first and third inputs, total reduction of $55 \%$ were obtained for the second input, respectively. These reductions are again obtained while maintaining the two outputs. With this decision of the DM for reducing the first and third inputs, the intermediate products, including the number of graduates and the tuition fees, which are also considered as the products of the education stage, have reached the values of 94630.5 and 61737.12 , respectively. Given that the products of the educational stage are used as inputs for the research stage, in this scenario, all the students have graduated and the entire existing tuition has been used as input for the research stage.

Note that in this study, it is assumed that the DM is more interested in optimizing consumed inputs in scenario (iii). The model could be easily setup for the case of the centralized DM interests for increasing the total amount of the produced outputs by imposing $\hat{\varphi}_{k} \geq 1$ values in model (3.4). 
TABLE 5. The results for the scenario (ii), $N^{\text {target }}=15$.

\begin{tabular}{llllllllll}
\hline \hline & $\hat{x}_{1}$ & $\hat{x}_{2}$ & $\hat{x}_{3}$ & $\hat{z}_{1}^{\text {out }}$ & $\hat{z}_{1}^{\text {in }}$ & $\hat{z}_{2}^{\text {out }}$ & $\hat{z}_{2}^{\text {in }}$ & $\hat{y}_{1}$ & $\hat{y}_{2}$ \\
\hline U1 & 0 & 0 & 0 & 0 & 0 & 0 & 0 & 0 & 0 \\
U2 & 75 & 8221 & 34 & 407 & 407 & 2636.2 & 2636.2 & 640.9 & 2923 \\
U3 & 0 & 0 & 0 & 0 & 0 & 0 & 0 & 0 & 0 \\
U4 & 35 & 4945 & 164 & 6716 & 9310 & 3146.3 & 7577.6 & 826.5 & 4868 \\
U5 & 0 & 0 & 0 & 0 & 0 & 0 & 0 & 0 & 0 \\
U6 & 64.12 & 7342.29 & 68.86 & 2099.23 & 407 & 2773.02 & 2636.2 & 640.9 & 2923 \\
U7 & 0 & 0 & 0 & 0 & 0 & 0 & 0 & 0 & 0 \\
U8 & 75 & 8221 & 34 & 407 & 1487 & 2636.2 & 5.07 & 7.14 & 3682 \\
U9 & 35 & 4945 & 164 & 6716 & 6977.81 & 3146.3 & 2358.55 & 557.49 & 4518.04 \\
U10 & 35 & 4945 & 164 & 6716 & 407 & 3146.3 & 2636.2 & 640.9 & 2923 \\
U11 & 75 & 8221 & 34 & 407 & 9327 & 2636.2 & 701.9 & 770.7 & 4879 \\
U12 & 75 & 8221 & 34 & 407 & 407 & 2636.2 & 2636.2 & 640.9 & 2923 \\
U13 & 0 & 0 & 0 & 0 & 0 & 0 & 0 & 0 \\
U14 & 35 & 4945 & 164 & 6716 & 407 & 3146.3 & 2636.2 & 640.9 & 2923 \\
U15 & 35 & 4945 & 164 & 6716 & 9310 & 3146.3 & 7577.6 & 826.5 & 4868 \\
U16 & 75 & 8221 & 34 & 407 & 407 & 2636.2 & 2636.2 & 640.9 & 2923 \\
U17 & 35 & 4945 & 164 & 6716 & 822.42 & 3146.3 & 1624.13 & 397.12 & 3214.95 \\
U18 & 35 & 4945 & 164 & 6716 & 1487 & 3146.3 & 5.07 & 7.14 & 3682 \\
U19 & 75 & 8221 & 34 & 407 & 9310 & 2636.2 & 7577.6 & 826.5 & 4868 \\
U20 & 75 & 8221 & 34 & 407 & 1487 & 2636.2 & 5.07 & 7.14 & 3682 \\
\hline Total & 834.12 & 99504.29 & 1454.86 & 51960.23 & 51960.23 & 43250.52 & 43249.8 & 8071.6 & 55800 \\
\hline Optimal & $\theta^{*}=0.338$ & $\theta^{*}=0.457$ & $\theta^{*}=0.427$ & - & - & - & - & $\varphi^{*}=1$ & $\varphi^{*}=1$ \\
\hline
\end{tabular}

TABLE 6 . The results for the scenario (iii), $\hat{\theta}_{1}=0.6$ and $\hat{\theta}_{3}=0.6$.

\begin{tabular}{llllllllll}
\hline \hline & $\hat{x}_{1}$ & $\hat{x}_{2}$ & $\hat{x}_{3}$ & $\hat{z}_{1}^{\text {out }}$ & $\hat{z}_{1}^{\text {in }}$ & $\hat{z}_{2}^{\text {out }}$ & $\hat{z}_{2}^{\text {in }}$ & $\hat{y}_{1}$ & $\hat{y}_{2}$ \\
\hline U1 & 0 & 0 & 0 & 0 & 0 & 0 & 0 & 0 & 0 \\
U2 & 0 & 0 & 0 & 0 & 0 & 0 & 0 & 0 & 0 \\
U3 & 90.6 & 7975.75 & 70.53 & 7784.51 & 8852.35 & 4971.62 & 7323.59 & 816.95 & 4868.02 \\
U4 & 0 & 0 & 0 & 0 & 0 & 0 & 0 & 0 & 0 \\
U5 & 35 & 4945 & 164 & 6716 & 9310 & 3146.2 & 7577.6 & 826.5 & 4868 \\
U6 & 35 & 4945 & 164 & 6716 & 9310 & 3146.2 & 7577.6 & 826.5 & 4868 \\
U7 & 0 & 0 & 0 & 0 & 0 & 0 & 0 & 0 & 0 \\
U8 & 170 & 12259 & 171 & 9310 & 1487 & 7577.6 & 5.07 & 7.14 & 3682 \\
U9 & 0 & 0 & 0 & 0 & 0 & 0 & 0 & 0 & 0 \\
U10 & 35 & 4945 & 164 & 6716 & 9310 & 3146.2 & 7577.6 & 826.4 & 4868 \\
U11 & 170 & 12259 & 171 & 9310 & 1487 & 7577.6 & 5.07 & 7.14 & 3682 \\
U12 & 0 & 0 & 0 & 0 & 0 & 0 & 0 & 0 & 0 \\
U13 & 170 & 12259 & 171 & 9310 & 9310 & 7577.6 & 7577.6 & 826.4 & 4868 \\
U14 & 35 & 4945 & 164 & 6716 & 9310 & 3146.3 & 7577.6 & 826.6 & 4868 \\
U15 & 35 & 4945 & 164 & 6716 & 8461.98 & 3146.3 & 625.01 & 686.4 & 4746.33 \\
U16 & 0 & 0 & 0 & 0 & 0 & 0 & 0 & 0 & 0 \\
U17 & 170 & 12259 & 171 & 9310 & 9310 & 7577.6 & 7577.6 & 826.5 & 4868 \\
U18 & 0 & 0 & 0 & 0 & 0 & 0 & 0 & 0 \\
U19 & 35 & 4945 & 164 & 6716 & 9310 & 3146.3 & 7577.6 & 826.5 & 4868 \\
U20 & 170 & 12259 & 171 & 9310 & 9172.17 & 7577.6 & 735.47 & 768.44 & 4745 \\
\hline Total & 1150.6 & 98940.75 & 2005.8 & 94630.5 & 94630.5 & 61737.12 & 61737.12 & 8071.4 & 55798.6 \\
\hline Optimal & $\theta^{*}=0.467$ & $\theta^{*}=0.454$ & $\theta^{*}=0.588$ & - & - & - & - & $\varphi^{*}=1$ & $\varphi^{*}=1$ \\
\hline
\end{tabular}


In all the scenarios mentioned above, the models attempted to improve the performance of the production system by reducing the total inputs and outputs. As shown in the example, the underwriting profit is unchanged, while the total investment profit is increased.

\section{SUMMARY AND CONCLUSION}

This paper attempted to introduce a procedure for efficient resource allocation in a centralized decisionmaking setting for a two-stage network system. In specific, this study aimed to evaluate the possibility of closing some DMUs in the interest of non-radial efficiency. Although different centralized approaches have been proposed in the literature, the possibility of closing observed DMUs is a novelty in DEA network system models. In this regard, three specific scenarios were discussed.

Scenario (i) aims to maximize the reduction in the total inputs and the increase in the total outputs, allowing for any existing DMU to be closed. This scenario has one phase. Any DMU that is closed in the first stage is closed in the second stage as well.

Scenario (ii) maximizes the reduction in the total inputs and the increase in the total outputs, setting a maximum number of surviving operating units. This maximum number is denoted by $N^{\text {target }}$, and is selected by the user. If it is set too low, there is the risk of obtaining an infeasible solution. However, if it is high enough, a solution similar to the first scenario would be found.

Scenario (iii) minimizes the number of active operating units, with a specific focus on reducing the total inputs and increasing the total outputs. The minimum reduction of total inputs corresponds to the parameter $\hat{\theta}$, while the maximum increase in total outputs corresponds to the parameter $\hat{\varphi}$, and are selected by the user. If either value is set too low or too high, the model may not yield a feasible solution.

This article provided a brief review of some basic network systems and their associated models. In realworld applications, some network production systems have a simple two-stage series structure. In this study, a new two-stage network DEA model was proposed, which can be used for centralized resource allocation with the possibility of downsizing of two-stage network structures. Finally, some numerical examples were used to illustrate the approach. Extending the provided approach for general network production systems could be considered as an interesting challenge in future studies. For future studies, this approach can be extended to incorporate different types of data such as quasi-fixed variables or undesirable outputs.

Acknowledgements. The authors are grateful to Nelson Maculan and two anonymous reviewers of this journal for their helpful comments and suggestions for improving the paper.

\section{REFERENCES}

[1] S. Ang, P. Liu and F. Yang, Intra-Organizational and inter-organizational resource allocation in two stage network systems. Omega 91 (2020) 102009.

[2] M. Asmild, J.C. Paradi and J.T. Pastor, Centralized resource allocation BCC models. Omega 37 (2009) 40-49.

[3] A.D. Athanassopoulos, Goal programming \& data envelopment analysis (GoDEA) for target-based multi-level planning: allocating central grants to the Greek local authorities. Eur. J. Oper. Res. 87 (1995) 535-550.

[4] A.D. Athanassopoulos, Decision support for target-based resource allocation of public services in multiunit and multilevel systems. Manage. Sci. 44 (1998) 173-187.

[5] S. Aviles-Sacoto, W.D. Cook, R. Imanirad and J. Zhu, Two-stage network DEA: when intermediate measures can be treated as outputs from the second stage. J. Oper. Res. Soc. 66 (2015) 1868-1877.

[6] R.D. Banker, A. Charnes and W.W. Cooper, Some models for estimating technical and scale inefficiencies in data envelopment analysis. Manage. Sci. 30 (1984) 1078-1092.

[7] J. Beasley, Allocating fixed costs and resources via data envelopment analysis. Eur. J. Oper. Res. 147 (2003) $198-216$.

[8] A. Charnes and W.W. Cooper, Programming with linear fractional functionals. Naval Res. Logistics Q. 9 (1962) $181-186$.

[9] A. Charnes, W.W. Cooper and E. Rhodes, Measuring the efficiency of decision making units. Eur. J. Oper. Res. 2 (1978) $429-444$.

[10] Y. Chen and J. Zhu, Measuring information technology's indirect impact on firm performance. Inf. Technol. Manage. 5 (2004) $9-22$.

[11] Y. Chen, W.D. Cook and J. Zhu, Deriving the DEA frontier for two-stage processes. Eur. J. Oper. Res. 202 (2010) $138-142$. 
[12] Y. Chen, J. Du, H. D. Sherman and J. Zhu, DEA model with shared resources and efficiency decomposition. Eur. J. Oper. Res. 207 (2010) 339-349.

[13] M.-C. Chen, M.-M. Yu and Y.-T. Ho, Using network centralized data envelopment analysis for shipping line resource allocation. Int. J. Environ. Sci. Technol. 15 (2018) 1777-1792.

[14] T. Ding, J. Yang, H. Wu, Y. Wen, C. Tan and L. Liang, Research performance evaluation of Chinese university: a nonhomogeneous network DEA approach. J. Manage. Sci. Eng. in press (2020). DOI: 10.1016/j.jmse.2020.10.003.

[15] J. Du, L. Liang, Y. Chen and G.-B. Bi, DEA-based production planning. Omega 38 (2010) 105-112.

[16] A. Emrouznejad, B.R. Parker and G. Tavares, Evaluation of research in efficiency and productivity: a survey and analysis of the first 30 years of scholarly literature in DEA. Soc.-Econ. Plann. Sci. 42 (2008) 151-157.

[17] A. Emrouznejad and G.-L. Yang, A survey and analysis of the first 40 years of scholarly literature in DEA: $1978-2016$. Soc.-Econ. Plan. Sci. 61 (2018) 4-8.

[18] L. Fang and C. Zhang, Resource allocation based on the DEA model. J. Oper. Res. Soc. 59 (2008) $1136-1141$.

[19] R. Färe, R. Grabowski, S. Grosskopf and S. Kraft, Efficiency of a fixed but allocatable input: a non-parametric approach. Econ. Lett. 56 (1997) 187-193.

[20] R. Färe, S. Grosskopf and G. Whittaker, Network dea. In: Modeling Data Irregularities and Structural Complexities in Data Envelopment Analysis. (2007) 209-240.

[21] M.J. Farrell, The measurement of productive efficiency. J. R. Stat. Soc.: Ser. A (General). 120 (1957) $253-281$.

[22] B. Golany, F. Phillips and J. Rousseau, Models for improved effectiveness based on DEA efficiency results. IIE Trans. 25 (1993) 2-10.

[23] B. Golany and E. Tamir, Evaluating efficiency-effectiveness-equality trade-offs: a data envelopment analysis approach. Manage. Sci. 41 (1995) 1172-1184.

[24] S.S. Hosseini, R. Kazemi Matin, M. Khunsiavash and Z. Moghadas, Measurement of productivity changes for general network production systems with stochastic data. Sädhanā 44 (2019) 72.

[25] C. Kao and S.-N. Hwang, Efficiency decomposition in two-stage data envelopment analysis: an application to non-life insurance companies in Taiwan. Eur. J. Oper. Res. 185 (2008) 418-429.

[26] P. Korhonen and M. Syrjänen, Resource allocation based on efficiency analysis. Manage. Sci. 50 (2004) 1134-1144.

[27] H.F. Lewis and T.R. Sexton, Network DEA: efficiency analysis of organizations with complex internal structure. Comput. Oper. Res. 31 (2004) 1365-1410.

[28] S. Lozano and G. Villa, Centralized resource allocation using data envelopment analysis. J. Prod. Anal. 22 (2004) $143-161$.

[29] S. Lozano and G. Villa, Centralized DEA models with the possibility of downsizing. J. Oper. Res. Soc. 56 (2005) 357-364.

[30] S. Lozano, G. Villa and R. Brännlund, Centralised reallocation of emission permits using DEA. Eur. J. Oper. Res. 193 (2009) $752-760$.

[31] S. Lozano, G. Villa and D. Canca, Application of centralised DEA approach to capital budgeting in Spanish ports. Comput. Ind. Eng. 60 (2011) 455-65.

[32] C. Mar-Molinero, D. Prior, M.-M. Segovia and F. Portillo, On centralized resource utilization and its reallocation by using DEA. Ann. Oper. Res. 221 (2014) 273-283.

[33] J.T. Pastor, J.L. Ruiz and I. Sirvent, An enhanced DEA Russell graph efficiency measure. Eur. J. Oper. Res. 115 (1999) 596-607.

[34] V. Patrizii, On network two stages variable returns to scale DEA models. Omega 97 (2020) 102084.

[35] A.E. Ripoll-Zarraga and S. Lozano, A centralised DEA approach to resource reallocation in Spanish airports. Ann. Oper. Res. 288 (2020) 701-732.

[36] L.M. Seiford and J. Zhu, Profitability and marketability of the top 55 US commercial banks. Manage. Sci. 45 (1999) $1270-1288$.

[37] J. Song, F. Wei, J. Chu, Q. Zhu and F. Yang, Allocating natural resource reduction amounts: a data envelopment analysis based-approach considering production technology heterogeneity. Expert Syst. 36 (2019) e12449.

[38] C.H. Wang, R.D. Gopal and S. Zionts, Use of data envelopment analysis in assessing information technology impact on firm performance. Ann. Oper. Res. 73 (1997) 191-213.

[39] G.-L. Yang, H. Fukuyama and Y.-Y. Song, Measuring the inefficiency of Chinese research universities based on a two-stage network DEA model. J. Inf. 12 (2018) 10-30.

[40] M.-M. Yu, C.-C. Chern and B. Hsiao, Human resource rightsizing using centralized data envelopment analysis: evidence from Taiwan's Airports. Omega 41 (2013) 119-130.

[41] Y. Zha and L. Liang, Two-stage cooperation model with input freely distributed among the stages. Eur. J. Oper. Res. 205 (2010) 332-338.

[42] T. Zhang, Y.-H. Chiu, Y. Li and T.-Y. Lin, Air pollutant and health-efficiency evaluation based on a dynamic network data envelopment analysis. Int. J. Environ. Res. Publ. Health 15 (2018) 2046.

[43] J. Zhu, Multi-factor performance measure model with an application to Fortune 500 companies. Eur. J. Oper. Res. 123 (2000) $105-124$. 


\section{Subscribe to Open (S2O) A fair and sustainable open access model}

This journal is currently published in open access with no charge for authors under a Subscribe-to-Open model (S2O). Open access is the free, immediate, online availability of research articles combined with the rights to use these articles fully in the digital environment.

$\mathrm{S} 2 \mathrm{O}$ is one of the transformative models that aim to move subscription journals to open access. Every year, as long as the minimum amount of subscriptions necessary to sustain the publication of the journal is attained, the content for the year is published in open access.

\section{Ask your library to support open access by subscribing to this $\mathrm{S} 2 \mathrm{O}$ journal.}

Please help to maintain this journal in open access! Encourage your library to subscribe or verify its subscription by contacting subscribers@edpsciences.org

We are thankful to our subscribers and sponsors for making it possible to publish the journal in open access, free of charge for authors. More information and list of sponsors: https://www.edpsciences.org/en/maths-s2o-programme 